\title{
Foliar mineral content of five shrub species with nutritional potential for small ruminants in semiarid regions in northeastern Mexico
}

\author{
Miguel Chávez Espinoza ${ }^{1}$ () Humberto González Rodríguez ${ }^{1 *}$ (i) Israel Cantú Silva $^{1}$ (i) \\ Mauricio Cotera Correa ${ }^{1}$ Andrés Eduardo Estrada Castillón ${ }^{1}$ Hugo $^{(-)}$Bernal Barragán $^{2}(\mathbb{D}$ \\ Marco Vinicio Gómez $\operatorname{Meza}^{3}$ (1)
}

${ }^{1}$ Universidad Autónoma de Nuevo León, Facultad de Ciencias Forestales, Carr. Nac. No. 85, km 145, Linares, Nuevo León 67700, México. E-mail: humberto.gonzalezrd@uanl.edu.mx. "Corresponding author.

${ }^{2}$ Universidad Autónoma de Nuevo León, Facultad de Agronomía, Col. Ex-Hacienda el Canadá, Escobedo, Nuevo León, México.

${ }^{3}$ Universidad Autónoma de Nuevo León, Facultad de Economía, Monterrey, Nuevo León, México.

ABSTRACT: This study was conducted to determine the monthly composition of the minerals $\mathrm{Ca}, \mathrm{P}, \mathrm{K}, \mathrm{Mg}, \mathrm{Cu}, \mathrm{Fe}, \mathrm{Mn}$ and $\mathrm{Zn}$ in five shrub species that are consumed by small ruminants in the semi-arid regions of northeastern Mexico. Leaves samples of shrub species; Celtis pallida, Croton suaveolens, Forestiera angustifolia, Guaiacum angustifolium and Parkinsonia aculeata were randomly collected during twelve consecutive months from July 2018 to June 2019 in two sites, located in Linares and Los Ramones counties, in the state of Nuevo Leon, Mexico. Mineral content was analyzed using an atomic absorption spectrophotometer, while phosphorus was measured by the colorimetry method. The Linares county showed the highest content of macro- and micro-minerals. Among species, C. pallida showed the highest values of macrominerals and P. aculeata content of micro-minerals was highest. Ca (total mean $\left.\left.=40.0 \mathrm{~g} \mathrm{~kg}^{-1} \mathrm{DM}\right), \mathrm{K}\left(15.4 \mathrm{~g} \mathrm{~kg}^{-1}\right), \mathrm{Mg}_{(3.7 \mathrm{~g} \mathrm{~kg}} \mathrm{g}^{-1}\right), \mathrm{Fe}(87.2$

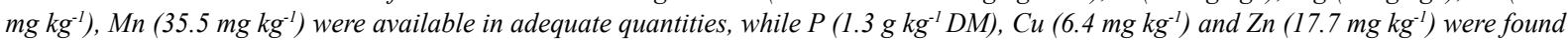
deficient. Shrub species in this study can be considered as a good source of Ca, K, Mg, Fe and Mn except in C. suaveolens. However, P, Cu and $\mathrm{Zn}$ must be supplemented.

Key words: grazing animals, leaves, nutritional requirements.

Conteúdo mineral foliar de cinco espécies de arbustos com potencial nutricional para pequenos ruminantes em regiões semiáridas do nordeste do México

RESUMO: $O$ presente estudo foi realizado com o intuito de determinar os teores dos minerais $\mathrm{Ca}, \mathrm{P}, \mathrm{K}, \mathrm{Mg}, \mathrm{Cu}, \mathrm{Fe}$, $\mathrm{Mn}$ e $\mathrm{Zn}$, ao longo do ano, em cinco espécies de arbustos consumidas por pequenos ruminantes nas regiões semiáridas do nordeste do México. Amostras de folhas de espécies arbustivas; Celtis pallida, Croton suaveolens, Forestiera angustifolia, Guaiacum angustifolium e Parkinsonia aculeata foram coletadas aleatoriamente durante doze meses consecutivos, de Julho de 2018 a Junho de 2019, em dois locais, nos municipios de Linares e Los Ramones, no estado de Nuevo Leon, México. O conteúdo mineral foi analisado através de um espectrofotômetro de absorção atômica, enquanto o fósforo foi medido pelo método da colorimetria. O município de Linares apresentou o maior conteúdo de macro e microminerais. Entre as espécies, C. pallida apresentou os maiores valores de macrominerais, enquanto o teor de microminerais foi maior na $P$. aculeata. Ca (média $\left.=40.0 \mathrm{~g} \mathrm{~kg}^{-1} \mathrm{MS}\right), \mathrm{K}\left(15.4 \mathrm{~g} \mathrm{~kg}^{-1}\right), \mathrm{Mg}\left(3.7 \mathrm{~g} \mathrm{~kg}^{-1}\right), \mathrm{Fe}\left(87.2 \mathrm{mg} \mathrm{kg} \mathrm{g}^{-1}\right), \mathrm{Mn}\left(35.5 \mathrm{mg} \mathrm{kg}^{-1}\right)$ estavam disponiveis em quantidades adequadas, enquanto $P\left(1.3 \mathrm{~g} \mathrm{~kg}^{-1} \mathrm{MS}\right), C u\left(6.4 \mathrm{mg} \mathrm{kg}^{-1}\right)$ e $\mathrm{Zn}\left(17.7 \mathrm{mg} \mathrm{kg}^{-1}\right)$ foram encontrados deficientes. As espécies arbustivas deste estudo podem ser consideradas uma boa fonte de Ca, $\mathrm{K}, \mathrm{Mg}$, Fe e Mn, exceto na espécie C. suaveolens. No entanto, $P$, Cu e Zn devem ser suplementados. Palavras-chave: animais em pastagem, folhas, necessidades nutricionais.

\section{INTRODUCTION}

The plant species distributed in the northeastern region of Mexico, which are categorized intoseveralgroups based on theirecologicaladaptations and forest use, constitute the type of vegetation namely, Tamaulipan Thornscrub. It covers about $125,000 \mathrm{~km}^{2}$ from the coastal plain of the Gulf of Mexico to the southern riviera of Texas in the United States of America (FOROUGHBAKHCH, 1992; FOROUGHBAKHCH et al., 2005; DOMÍNGUEZGÓMEZ et al., 2012). The vegetation of this area, 
consists of small trees and shrubs of almost 60 woody species (LUDWIG et al., 1975; DOMÍNGUEZGÓMEZ et al., 2012), many of them important in forestry and silvopastoral production (wood, poles, firewood, fodder, charcoal, medicine, etc.), which are the most common known uses as documented by ESTRADA-CASTILLÓN et al. (2018). Livestock production based on grazing occurs mainly in arid and semi-arid regions, on land traditionally considered unsuitable for crop production, containing woody and shrubs plants relevant as potential fodder source (ESTELL et al., 2012), which contain satisfactory levels of protein and minerals for grazing ruminants, while maintaining or improving grassland availability (RAMÍREZ-ORDUÑA et al., 2005; GUERRERO-CERVANTES et al., 2012; HABIB et al., 2016; DERERO \& KITAW, 2018). Many trees and shrubs of leguminous species of the semi-arid regions are used as feed especially due to the behavior in extensive grazing by goats and sheep (SANON et al., 2007) and also by wild ruminants, since shrub species remain green and with a relatively high nutrient content throughout the year (GUERRERO-CERVANTES et al., 2008). The shrubs Celtis pallida Torr. (Family Ulmaceae), Croton suaveolens Torr. (Family Euphorbiaceae), Forestiera angustifolia Torr. (Family Oleaceae), Guaiacum angustifolium Engelm. (Family Zygophyllaceae) and Parkinsonia aculeata L. (Family Leguminosae) apparently do not contain in their leaves compounds with negative side effects (SCHINDLER et al., 2003; SALEM et al., 2014). However, sometimes they may have imbalanced mineral concentrations, knowledge of their mineral composition and correct supplementation is crucial (HABIB et al., 2016), since they are consumed in different percentages throughout the year (MEYER et al., 1984; RAMÍREZ et al., 1997).

The present study hypothesized that leaves of native shrubs of northeastern Mexico represent an important feed and mineral resource for small ruminants such as goats, sheep and white-tailed deer, under extensive production systems when pastures have poor quality due to seasonal variation. The leaves of these plants may be an alternative that has not been studied sufficiently and could represent a valuable emergency feed resource.

The aim of this study was to determine the monthly foliar concentration of $\mathrm{Ca}, \mathrm{P}, \mathrm{K}, \mathrm{Mg}, \mathrm{Cu}$, $\mathrm{Fe}, \mathrm{Mn}$ and $\mathrm{Zn}$ of five native shrubs, throughout the year at two study sites in northeastern Mexico and to estimate if they have the potential to meet the mineral requirements of small ruminants.

\section{MATERIALS AND METHODS}

The studied vegetation was sampled at two sites located in the state of Nuevo Leon, Mexico, in the municipalities of Linares and Los Ramones, whose physical characteristics are: Site 1, Linares; the area of study was established within the Experimental Campus of the College of Forest Sciences of the Autonomous University of Nuevo Leon, located in Linares municipality $\left(24^{\circ} 47^{\prime} 45^{\prime \prime}\right.$ $\mathrm{N}$; $99^{\circ} 32^{\prime} 31^{\prime \prime} \mathrm{W} ; 350$ meters above sea level). The climate is subtropical and semi-arid with a warm summer (GONZÁLEZ-RODRÍGUEZ et al., 2004). The monthly average ambient temperature recorded during the experimental period ranged from $14.1{ }^{\circ} \mathrm{C}$ in January to $30.4{ }^{\circ} \mathrm{C}$ in August. Annual registered rainfall was $554 \mathrm{~mm}$.

Site 2, Los Ramones; the area of study was established in "El Abuelo Ranch" in Los Ramones municipality, with an area of 100 ha and an altitude of 200 meters above sea level. The geographical location is $25^{\circ} 39^{\prime} 46^{\prime \prime} \mathrm{N}$; $99^{\circ} 27^{\prime} 51^{\prime \prime} \mathrm{W}$. Climate is semi-arid with warm summer (GONZÁLEZ-RODRÍGUEZ et al., 2004). The average monthly ambient temperature during the study varied from $14.3{ }^{\circ} \mathrm{C}$ in January to $31.5^{\circ} \mathrm{C}$ in August. The annual recorded rainfall was approximately $667 \mathrm{~mm}$.

Experimental plots $\left(\begin{array}{lllll}50 & \mathrm{~m} & \mathrm{x} & 50 & \mathrm{~m}\end{array}\right)$ representative of each study site, were established without disturbance. Leaf tissue samples from five randomly selected individuals representative of each of the five species of shrub plants in this study were sampled: Celtis pallida, Croton suaveolens, Forestiera angustifolia, Guaiacum angustifolium and Parkinsonia aculeata were collected at a height of $1 \mathrm{~m}$ to $1.5 \mathrm{~m}$ from the ground, at monthly intervals from July 2018 to June 2019.

The collected material was dried for 24 $\mathrm{h}$ at $55{ }^{\circ} \mathrm{C}$ in a forced air oven (Felisa ${ }^{\circledR}$, Model FE292AD, Mexico), in the Laboratory of Chemistry and Plant Physiology of the College of Forest Sciences, the leaves were separated from the branches. Once the foliar samples were identified by month, species and site, they were ground in a Thomas Willey mill (Thomas Scientific Apparatus, Model 3383) using a No. $60 \mathrm{mesh}$ ( $1 \mathrm{~mm} \times 1 \mathrm{~mm}$ ), and the ground samples were stored in plastic bags.

Dry matter content (DM) of the leaves samples was determined in quintuplicate, weighing $1.0 \mathrm{~g}$ of sample, which was dried at $100{ }^{\circ} \mathrm{C}$ for $24 \mathrm{~h}$ in a forced air oven $\left(\right.$ Felisa $^{\circledR}$, Model FE292AD, Mexico). The organic matter (MO, \%) and ash $(\%)$ content was determined by incinerating the 
sample at $550{ }^{\circ} \mathrm{C}$ for $3 \mathrm{~h} 30 \mathrm{~min}$ by using a furnace (Thermo scientific, Model F48010, U.S.A.). The ashes were used to determined macro-minerals (Ca, $\mathrm{Mg}$ and $\mathrm{K} ; \mathrm{g} \mathrm{kg}^{-1} \mathrm{DM}$ ) and micro-minerals $\left(\mathrm{Cu}, \mathrm{Fe}, \mathrm{Mn}\right.$ and $\left.\mathrm{Zn} ; \mathrm{mg} \mathrm{kg}^{-1} \mathrm{DM}\right)$ by atomic absorption spectrophotometry (AOAC, 1997), using a spectrophotometer (model PinAAcle 900F, Perkin Elmer). The phosphorus content was measured by the colorimetry method (AOAC, 1997), using a UV-Visible spectrophotometer (model Lambda 1A, Perkin-Elmer).

To measure the influence of temperature and precipitation at each site, automated HOBO sensors (HOBO Pro Temp / RH Series, Forestry Suppliers, Inc., Jackson, MS, USA) were installed to record hourly environmental variables such as temperature of the air $\left({ }^{\circ} \mathrm{C}\right)$ and relative humidity $(\%)$. The daily amount of rainfall (mm) was quantified using a Davis brand automated rain gauge, connected to a HOBO Event Onset recorder.

\section{Statistical analysis}

The statistical analysis of variance and mean comparison of the observed data for each mineral were analyzed by a completely randomized design with a factorial arrangement, with 5 species, two sites and 12 months as factors, and considering double and triple interactions. Statistical analyses were performed by using the SPSS statistical analysis software (SPSS for Windows, Version 22.0, Chicago, IL, USA).

\section{RESULTS}

Macro (Ca, P, K and $\mathrm{Mg}$ ) and trace minerals $(\mathrm{Cu}, \mathrm{Fe}, \mathrm{Mn}$ and $\mathrm{Zn})$ were significantly different between sites (except Fe), months and species. The double and triple interactions were also significant. Rainfall, relative humidity and temperature recorded during the study are shown in table 1 . None of the quantified minerals were significantly correlated, with rainfall, relative humidity and temperature recorded during the period of study.

The $\mathrm{Ca}$ content was higher in Linares, compared to Los Ramones. All species showed the highest $\mathrm{Ca}$ content in the month of June. C. pallida had the highest concentrations, while $C$. suaveolens had the lowest values (Table 2). Los Ramones and the species $P$. aculeata and G. angustifolium showed higher and lower concentrations in $\mathrm{P}$, respectively (Table 3). The highest concentration of $\mathrm{P}$ was recorded for samples collected in March, while those harvested in July and August were the lowest. Potassium content was higher in leaf samples from Los Ramones site. Regardless of location, the sampled leaves showed the highest $\mathrm{K}$ content during April and the lowest level in August. C. suaveolens had the highest K content; $G$. angustifolium showed the minimum (Table 4). The magnesium content was higher in leaf samples of the species in Los Ramones. All shrubs showed higher $\mathrm{Mg}$ values in November and lower in December. $C$. pallida generally had the highest $\mathrm{Mg}$ content, while $P$. aculeata had the lowest (Table 5).

Table 1 - Monthly mean temperature $\left(\mathrm{T},{ }^{\circ} \mathrm{C}\right)$, relative humidity $(\mathrm{RH}, \%)$ and rainfall $(\mathrm{mm})$ registered at the research sites

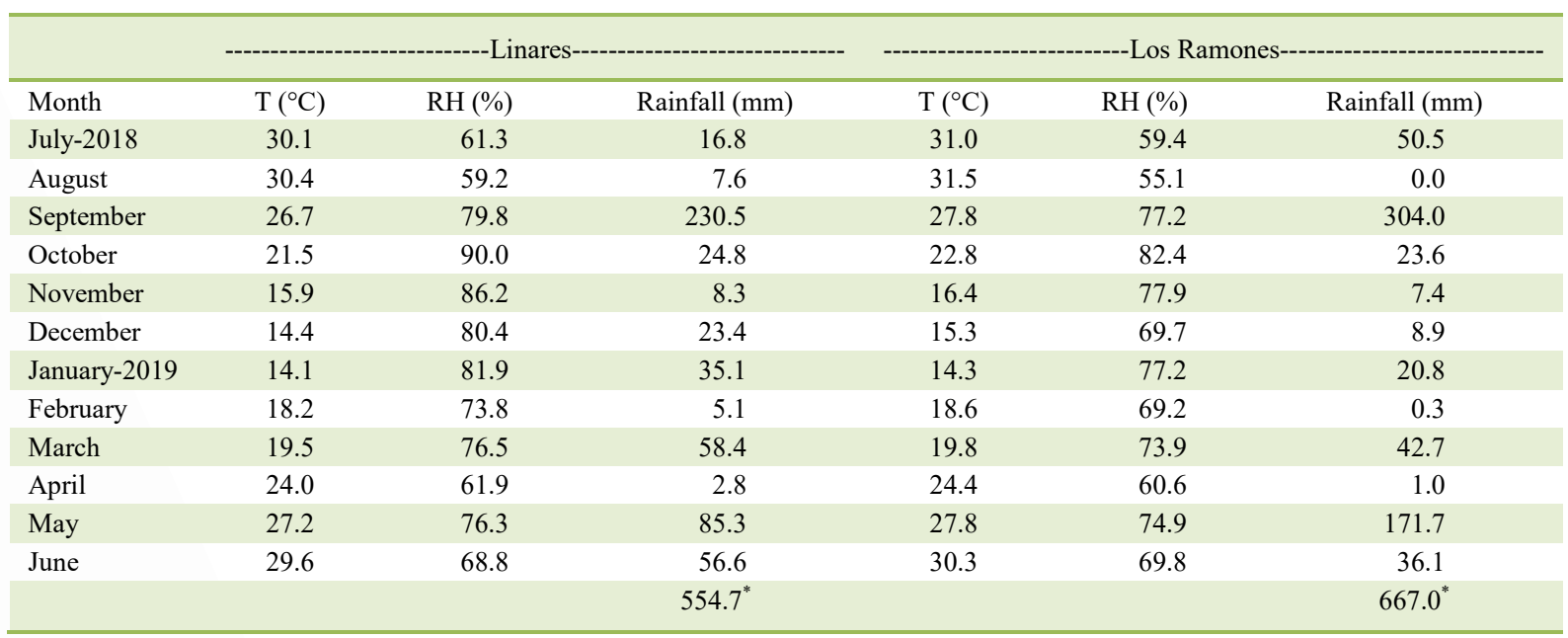

*Accumulated rainfall (mm). 
Table 2 - Monthly foliar Ca content ( $\mathrm{g} \mathrm{kg}^{-1}$ dry matter) in five shrubs species from northeastern Mexico sampled at two study sites from July 2018 to June 2019. Data represents the mean $(n=5)$.

\begin{tabular}{|c|c|c|c|c|c|c|c|c|c|c|c|c|}
\hline \multirow{2}{*}{$\begin{array}{l}\text { Site } \\
\text { Month }\end{array}$} & \multicolumn{5}{|c|}{----------------------------Linares---------------------------- } & \multicolumn{5}{|c|}{ 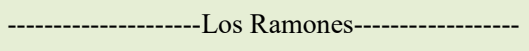 } & \multirow[b]{2}{*}{ Mean } & \multirow[b]{2}{*}{ SEM } \\
\hline & C.p. & C.s. & $F . a$. & G. $a$. & $P . a$. & C. $p$. & C.s. & $F . a$. & G. $a$. & P. a. & & \\
\hline July & 62.5 & 11.2 & 12.8 & 45.0 & 25.0 & 40.3 & 11.4 & 8.7 & 31.5 & 16.2 & 26.5 & 0.9 \\
\hline August & 52.5 & 9.7 & 10.6 & 45.8 & 19.5 & 48.8 & 11.2 & 16.3 & 53.4 & 24.8 & 29.3 & 0.9 \\
\hline September & 113.3 & 13.3 & 16.7 & 83.5 & 34.2 & 94.5 & 15.1 & 16.9 & 50.9 & 27.5 & 46.6 & 0.9 \\
\hline October & 73.0 & 19.9 & 19.4 & 89.3 & 26.4 & 80.5 & 15.8 & 15.2 & 78.9 & 18.5 & 43.7 & 0.9 \\
\hline November & 86.4 & 16.8 & 19.6 & 91.7 & 27.0 & 101.6 & 14.7 & 16.0 & 63.2 & 15.9 & 45.3 & 0.9 \\
\hline December & 58.5 & 22.6 & 24.9 & 61.4 & 36.0 & 65.0 & 19.5 & 19.5 & 53.2 & 16.2 & 37.7 & 0.9 \\
\hline January & 64.6 & 25.3 & 24.7 & 52.6 & 30.0 & 62.6 & 22.6 & 23.2 & 54.9 & 17.9 & 37.8 & 0.9 \\
\hline February & 64.6 & 25.1 & 17.2 & 58.1 & 24.1 & 57.8 & 17.4 & 22.5 & 52.4 & 17.0 & 35.6 & 0.9 \\
\hline March & 91.2 & 20.5 & 23.3 & 52.3 & 17.5 & 73.6 & 33.5 & 47.0 & 54.2 & 32.4 & 44.6 & 0.9 \\
\hline April & 80.7 & 19.7 & 10.9 & 40.5 & 7.3 & 65.2 & 13.6 & 12.7 & 58.1 & 7.4 & 31.6 & 0.9 \\
\hline May & 132.6 & 19.0 & 13.0 & 53.2 & 18.9 & 130.9 & 14.0 & 12.6 & 71.7 & 19.4 & 48.5 & 0.9 \\
\hline June & 120.4 & 23.5 & 18.1 & 77.2 & 37.6 & 121.8 & 16.2 & 15.9 & 79.1 & 20.1 & 53.0 & 0.9 \\
\hline Mean & 83.4 & 18.9 & 17.6 & 62.6 & 25.3 & 78.6 & 17.1 & 18.9 & 58.5 & 19.4 & 40.0 & \\
\hline SEM & 0.9 & 0.8 & 0.8 & 0.9 & 0.8 & 0.9 & 0.8 & 0.8 & 0.8 & 0.8 & & \\
\hline Site mean & \multicolumn{5}{|c|}{------------------------------41.5------------------------------ } & \multicolumn{5}{|c|}{----------------------------38.5------------------------- } & & \\
\hline SEM & \multicolumn{5}{|c|}{----------------------------0.3---------------------------- } & \multicolumn{5}{|c|}{--------------------------0.3-------------------------' } & & \\
\hline Factors & \multicolumn{2}{|c|}{---Month (A)---- } & Site (B) & \multicolumn{2}{|c|}{--Species (C)--- } & $\mathrm{A} \times \mathrm{B}$ & \multirow{2}{*}{\multicolumn{2}{|c|}{------A x C----- }} & \multicolumn{2}{|c|}{------B x C------ } & \multicolumn{2}{|c|}{$---A$ x B x C---- } \\
\hline $\mathrm{p}$ & \multicolumn{2}{|c|}{-------- ${ }^{*} *{ }^{*}---------$} & $* * *$ & \multicolumn{2}{|c|}{-1--- } & *** & & & \multicolumn{2}{|c|}{-------" ${ }^{* * *}-------$} & \multicolumn{2}{|c|}{ - } \\
\hline
\end{tabular}

C. p.; Celtis pallida, C. s.; Croton suaveolens, F. a.; Forestiera angustifolia, G. a.; Guaiacum angustifolium, P. a.; Parkinsonia aculeata.

SEM, standard error of the mean.

Significant at 0.001 level, ${ }^{* * *}$ significant at 0.01 level.

Table 3 - Monthly foliar P content ( $\mathrm{g} \mathrm{kg}^{-1}$ dry matter) in five shrubs species from northeastern Mexico sampled at two study sites from July 2018 to June 2019. Data represents the mean $(n=5)$.

\begin{tabular}{|c|c|c|c|c|c|c|c|c|c|c|c|c|}
\hline \multirow{2}{*}{$\begin{array}{l}\text { Site } \\
\text { Month }\end{array}$} & \multicolumn{5}{|c|}{ 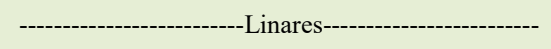 } & \multicolumn{7}{|c|}{ 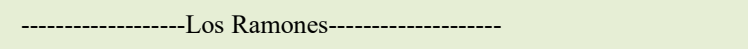 } \\
\hline & C.p. & C.s. & F. $a$. & G. $a$. & P. $a$. & C.p. & C.s. & F. $a$. & G. $a$. & P. a. & Mean & SEM \\
\hline July & 1.06 & 1.09 & 0.94 & 0.79 & 1.42 & 1.33 & 1.35 & 1.00 & 0.78 & 1.54 & 1.13 & 0.01 \\
\hline August & 0.84 & 1.30 & 0.79 & 0.97 & 1.36 & 1.09 & 0.92 & 0.91 & 0.66 & 1.21 & 1.00 & 0.01 \\
\hline September & 0.99 & 1.24 & 0.84 & 0.78 & 1.07 & 1.19 & 1.65 & 1.09 & 0.76 & 1.88 & 1.15 & 0.01 \\
\hline October & 1.46 & 1.64 & 1.08 & 0.95 & 2.00 & 1.48 & 1.37 & 1.19 & 0.81 & 2.00 & 1.40 & 0.01 \\
\hline November & 1.43 & 1.28 & 0.98 & 0.89 & 1.59 & 1.17 & 1.05 & 1.01 & 0.82 & 1.63 & 1.18 & 0.01 \\
\hline December & 1.21 & 1.29 & 0.90 & 0.82 & 1.25 & 1.18 & 1.04 & 1.26 & 0.90 & 1.74 & 1.16 & 0.01 \\
\hline January & 1.18 & 1.39 & 1.08 & 0.80 & 1.49 & 1.19 & 1.06 & 1.08 & 0.83 & 1.54 & 1.16 & 0.01 \\
\hline February & 1.35 & 1.52 & 1.64 & 0.71 & 1.49 & 1.28 & 1.25 & 1.75 & 0.72 & 1.60 & 1.33 & 0.01 \\
\hline March & 0.87 & 2.02 & 1.38 & 1.77 & 2.46 & 1.57 & 1.43 & 1.63 & 1.61 & 2.80 & 1.75 & 0.01 \\
\hline April & 1.73 & 1.76 & 1.03 & 1.50 & 2.52 & 1.80 & 1.77 & 1.39 & 1.09 & 2.02 & 1.66 & 0.01 \\
\hline May & 1.34 & 1.76 & 0.99 & 1.06 & 1.97 & 1.59 & 1.99 & 1.14 & 0.80 & 1.77 & 1.44 & 0.01 \\
\hline June & 1.20 & 1.49 & 1.04 & 0.95 & 1.39 & 1.46 & 1.26 & 1.00 & 0.70 & 1.75 & 1.22 & 0.01 \\
\hline Mean & 1.22 & 1.48 & 1.06 & 1.00 & 1.67 & 1.36 & 1.34 & 1.20 & 0.87 & 1.79 & 1.30 & \\
\hline SEM & 0.01 & 0.01 & 0.01 & 0.01 & 0.01 & 0.01 & 0.01 & 0.01 & 0.01 & 0.01 & & \\
\hline Site mean & \multicolumn{5}{|c|}{ 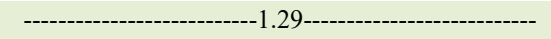 } & \multicolumn{7}{|c|}{-----------------------------1.31-------------------------- } \\
\hline SEM & \multicolumn{5}{|c|}{-----------------------------0.01----------------------------- } & \multicolumn{5}{|c|}{----------------------------0.01------------------------- } & & \\
\hline Factors & \multicolumn{2}{|c|}{ Month (A) } & Site (B) & \multicolumn{2}{|c|}{ Species (C) } & $\mathrm{A} \times \mathrm{B}$ & ------- & C----- & \multirow{2}{*}{\multicolumn{2}{|c|}{----B x C----- }} & \multirow{2}{*}{\multicolumn{2}{|c|}{$--A \times{ }_{* * *}$}} \\
\hline $\mathrm{p}$ & -------- & -1--- & $* *$ & & - & *** & & & & & & \\
\hline
\end{tabular}

C. p.; Celtis pallida, C. s.; Croton suaveolens, F. a.; Forestiera angustifolia, G. a.; Guaiacum angustifolium, P. a.; Parkinsonia aculeata. SEM, standard error of the mean.

Significant at 0.001 level, ${ }^{* *}$ significant at 0.01 level. 
Foliar mineral content of five shrub species with nutritional potential for small ruminants in semiarid regions in northeastern Mexico. 5

Table 4 - Monthly foliar $\mathrm{K}$ content ( $\mathrm{g} \mathrm{kg}^{-1}$ dry matter) in five shrubs species from northeastern Mexico sampled at two study sites from July 2018 to June 2019. Data represents the mean $(n=5)$

\begin{tabular}{|c|c|c|c|c|c|c|c|c|c|c|c|c|}
\hline \multirow{2}{*}{$\begin{array}{l}\text { Site } \\
\text { Month }\end{array}$} & \multicolumn{5}{|c|}{--------------------------'Linares-------------------------- } & \multicolumn{7}{|c|}{ 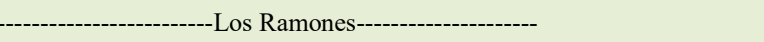 } \\
\hline & C. $p$. & C. s. & F. a. & G. $a$. & P. $a$. & C. $p$. & C.s. & F. a. & G. $a$. & P. a. & Mean & SEM \\
\hline July & 13.9 & 14.8 & 24.4 & 5.0 & 11.9 & 20.3 & 21.0 & 16.1 & 6.1 & 11.8 & 14.5 & 0.4 \\
\hline August & 14.4 & 12.2 & 13.7 & 5.8 & 13.3 & 16.6 & 16.0 & 15.6 & 6.6 & 11.7 & 12.6 & 0.4 \\
\hline September & 13.4 & 13.3 & 13.8 & 4.6 & 11.3 & 15.9 & 20.9 & 16.0 & 7.7 & 12.2 & 12.9 & 0.4 \\
\hline October & 22.5 & 25.9 & 16.3 & 4.1 & 13.7 & 14.1 & 23.7 & 21.7 & 6.9 & 14.7 & 16.4 & 0.4 \\
\hline November & 16.5 & 19.9 & 11.8 & 4.1 & 12.1 & 12.7 & 24.0 & 23.0 & 7.1 & 16.5 & 14.8 & 0.4 \\
\hline December & 22.5 & 23.7 & 13.9 & 4.6 & 11.4 & 17.3 & 23.1 & 21.5 & 10.0 & 17.8 & 16.6 & 0.4 \\
\hline January & 19.6 & 18.1 & 18.5 & 8.9 & 14.9 & 14.2 & 15.4 & 18.0 & 12.4 & 15.7 & 15.6 & 0.4 \\
\hline February & 22.6 & 15.0 & 19.4 & 8.7 & 12.9 & 17.0 & 15.7 & 17.4 & 8.6 & 17.0 & 15.4 & 0.4 \\
\hline March & 19.7 & 19.7 & 20.9 & 11.2 & 14.9 & 14.2 & 18.2 & 17.5 & 14.1 & 16.9 & 16.7 & 0.4 \\
\hline April & 19.8 & 22.9 & 18.2 & 11.2 & 15.8 & 18.4 & 23.2 & 22.8 & 11.1 & 14.3 & 17.8 & 0.4 \\
\hline May & 18.0 & 17.6 & 16.4 & 7.8 & 14.0 & 19.4 & 20.7 & 15.0 & 10.5 & 12.8 & 15.2 & 0.4 \\
\hline June & 21.8 & 20.9 & 21.5 & 7.5 & 15.2 & 17.5 & 21.7 & 18.9 & 9.2 & 12.0 & 16.6 & 0.4 \\
\hline Mean & 18.7 & 18.6 & 17.4 & 7.0 & 13.5 & 16.5 & 20.3 & 18.6 & 9.2 & 14.5 & 15.4 & \\
\hline SEM & 0.3 & 0.3 & 0.3 & 0.3 & 0.3 & 0.3 & 0.3 & 0.3 & 0.3 & 0.3 & & \\
\hline Site mean & \multicolumn{12}{|c|}{-------------------------------15.0----------------------------- } \\
\hline SEM & \multicolumn{5}{|c|}{----------------------------0.1----------------------------- } & \multicolumn{7}{|c|}{--------------------------------0.1 1---------------------------- } \\
\hline Factors & \multicolumn{2}{|c|}{ Month (A) } & Site (B) & \multicolumn{2}{|c|}{ Species (C) } & $\mathrm{A} \times \mathrm{B}$ & \multirow{2}{*}{\multicolumn{2}{|c|}{$\begin{array}{c}-----A x \text { x C----- } \\
\text { *** } \\
-------\end{array}$}} & \multicolumn{2}{|c|}{------B x C------ } & \multicolumn{2}{|c|}{$--\mathrm{A}$ x B x C-- } \\
\hline $\mathrm{p}$ & \multicolumn{2}{|c|}{-------" } & *** & \multicolumn{2}{|c|}{---------"** -------- } & *** & & & \multicolumn{2}{|c|}{-------'***----- } & \multicolumn{2}{|c|}{------" ${ }^{* *}------$} \\
\hline
\end{tabular}

C. p.; Celtis pallida, C. s.; Croton suaveolens, F. a.; Forestiera angustifolia, G. a.; Guaiacum angustifolium, P. a.; Parkinsonia aculeata. SEM, standard error of the mean.

${ }^{* * *}$ Significant at 0.001 level, ${ }^{* *}$ significant at 0.01 level.

Table 5 - Monthly foliar Mg content ( $\mathrm{g} \mathrm{kg}^{-1}$ dry matter) in five shrubs species from northeastern Mexico sampled at two study sites from July 2018 to June 2019. Data represents the mean $(n=5)$

\begin{tabular}{|c|c|c|c|c|c|c|c|c|c|c|c|c|}
\hline \multirow{2}{*}{$\begin{array}{l}\text { Site } \\
\text { Month }\end{array}$} & \multicolumn{5}{|c|}{----------------------------Linares-------------------------- } & \multicolumn{7}{|c|}{------------------------Los Ramones------------------- } \\
\hline & C.p. & C.s. & $F . a$. & G. $a$. & $P . a$. & C. $p$. & C. $s$. & $F . a$. & G. $a$. & $P . a$ & Mean & SEM \\
\hline July & 6.5 & 2.7 & 2.4 & 4.4 & 3.3 & 6.8 & 3.8 & 2.7 & 3.9 & 2.2 & 3.9 & 0.1 \\
\hline August & 6.0 & 2.3 & 2.4 & 4.6 & 2.0 & 7.9 & 3.5 & 3.3 & 4.5 & 1.9 & 3.9 & 0.1 \\
\hline September & 7.2 & 2.7 & 2.7 & 5.8 & 2.8 & 8.1 & 3.9 & 2.8 & 4.3 & 2.1 & 4.2 & 0.1 \\
\hline October & 6.7 & 4.4 & 3.2 & 5.5 & 2.2 & 8.1 & 3.9 & 3.0 & 4.8 & 2.4 & 4.4 & 0.1 \\
\hline November & 5.9 & 4.2 & 3.7 & 5.8 & 2.5 & 8.4 & 3.3 & 3.3 & 5.1 & 2.2 & 4.4 & 0.1 \\
\hline December & 3.4 & 2.5 & 2.5 & 4.1 & 1.7 & 7.1 & 2.4 & 2.3 & 3.4 & 1.5 & 3.1 & 0.1 \\
\hline January & 5.8 & 2.5 & 2.0 & 2.3 & 1.6 & 6.8 & 2.6 & 3.4 & 3.8 & 1.9 & 3.3 & 0.1 \\
\hline February & 4.6 & 3.3 & 1.8 & 3.4 & 1.5 & 5.8 & 2.7 & 3.1 & 3.7 & 1.4 & 3.1 & 0.1 \\
\hline March & 5.8 & 2.5 & 1.7 & 3.0 & 1.6 & 6.9 & 3.4 & 2.5 & 2.3 & 1.8 & 3.1 & 0.1 \\
\hline April & 5.5 & 2.7 & 1.5 & 2.3 & 1.5 & 6.9 & 3.9 & 2.4 & 3.4 & 1.5 & 3.2 & 0.1 \\
\hline May & 6.2 & 2.8 & 1.2 & 2.7 & 1.6 & 8.2 & 3.3 & 1.6 & 3.7 & 2.1 & 3.3 & 0.1 \\
\hline June & 6.3 & 2.8 & 2.4 & 3.9 & 2.0 & 8.0 & 3.9 & 2.7 & 4.7 & 2.3 & 3.9 & 0.1 \\
\hline Mean & 5.8 & 2.9 & 2.3 & $4.0^{*}$ & $2.0^{*}$ & 7.4 & 3.4 & 2.8 & $4.0^{*}$ & $1.9^{*}$ & 3.7 & \\
\hline SEM & 0.1 & 0.1 & 0.1 & 0.1 & 0.1 & 0.1 & 0.1 & 0.1 & 0.1 & 0.1 & & \\
\hline Site mean & \multicolumn{5}{|c|}{------------------------------3.4------------------------------ } & \multicolumn{7}{|c|}{------------------------------3.9-------------------------------' } \\
\hline SEM & \multicolumn{5}{|c|}{-----------------------------0.0---------------------------- } & \multicolumn{7}{|c|}{ 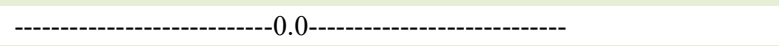 } \\
\hline Factors & \multicolumn{2}{|c|}{ Month (A) } & Site (B) & \multicolumn{2}{|c|}{ Species (C) } & $\mathrm{A} \times \mathrm{B}$ & \multicolumn{2}{|c|}{------A x C------ } & ------ & C----- & \multicolumn{2}{|c|}{---A x B x C-- } \\
\hline $\mathrm{p}$ & \multicolumn{2}{|c|}{---------" ${ }^{* * *}---------$} & $* * *$ & \multicolumn{2}{|c|}{--------" ${ }^{* * *}$--------- } & $* * *$ & \multicolumn{2}{|c|}{---------- ${ }^{* * *}$--------- } & \multicolumn{2}{|c|}{--- ${ }^{* * *}--------$} & \multicolumn{2}{|c|}{-------" ${ }^{* * *}-------$} \\
\hline
\end{tabular}

C. p.; Celtis pallida, C. s.; Croton suaveolens, F. a.; Forestiera angustifolia, G. a.; Guaiacum angustifolium, P. a.; Parkinsonia aculeata.

SEM, standard error of the mean.

**** Significant at 0.001 level, ${ }^{* *}$ significant at 0.01 level. ${ }^{*}$ Site factor were not significant between same shrub species

Ciência Rural, v.50, n.10, 2020. 
The $\mathrm{Cu}$ content (Table 6) was higher in leaf samples from the site of Linares compared to Los Ramones. Shrubs sampled in March showed the highest $\mathrm{Cu}$ content of the year, while concentrations were lower in samples collected in January. P. aculeata and C. suaveolens had a similar $\mathrm{Cu}$ content and were higher than $C$. pallida. The Fe content of $C$. suaveolens exhibited the highest values (Table 7), January leaf samples had the highest while July showed the lowest concentrations. Manganese content was higher in $P$. aculeata followed by $F$. angustifolia, in addition, a higher Mn content was recorded in foliar samples of the Linares site than in the samples of shrubs collected in Los Ramones, which were generally lower. During August, lower values were recorded, while in January they were higher (Table 8). The zinc content was highest in March, while the lowest was recorded in September among the species studied. The highest level of $\mathrm{Zn}$ was reported in $F$. angustifolia, while $C$. pallida recorded the lowest levels. Higher concentrations of $\mathrm{Zn}$ were observed in foliar samples at Los Ramones site (Table 9).

\section{DISCUSSION}

The calcium content was higher in Linares, compared to Los Ramones, this contrasts with that reported by DOMÍNGUEZ-GÓMEZ et al. (2014), also $C$. pallida had highest concentrations in our study. It seems that regardless of location, species or months, the foliar content of $\mathrm{Ca}$ (total mean $=40.0$ $\mathrm{g} \mathrm{kg}^{-1}$ ) exceeded the requirement recommended by NRC (2007) for adult white-tailed deer, sheep and goats $\left(5.1,3.0,5.3 \mathrm{~g} \mathrm{~kg}^{-1} \mathrm{DM}\right.$ for $\mathrm{Ca}$, severally). Probably the high $\mathrm{Ca}$ content in these sites is due to the fact that the soil, is a calcisol with high $\mathrm{pH}$ (CANTÚ et al., 2018). The high Ca concentrations contained in soil will eventually be reflected in the leaves of the plants (FAN et al., 2019). High calcium content in plants consumed by ruminants in extensive grazing in semi-arid regions were also reported by HABIB et al. (2016) in Pakistan and by GUERREROCERVANTES et al. (2012) in northern Mexico.

In the present study regardless of shrub species, sampling month or site the content of $\mathrm{P}$ (total mean $=1.3 \mathrm{~g} \mathrm{~kg}^{-1} \mathrm{DM}$ ), was insufficient to meet the

Table 6 - Monthly foliar $\mathrm{Cu}$ content ( $\mathrm{mg} \mathrm{kg}^{-1}$ dry matter) in five shrubs species from northeastern Mexico sampled at two study sites from July 2018 to June 2019. Data represents the mean $(n=5)$.

\begin{tabular}{|c|c|c|c|c|c|c|c|c|c|c|c|c|}
\hline \multirow{2}{*}{$\begin{array}{l}\text { Site } \\
\text { Month }\end{array}$} & \multicolumn{5}{|c|}{-----------------------------Linares------------------------- } & \multicolumn{7}{|c|}{-----------------------Los Ramones---------------------- } \\
\hline & C.p. & C. $s$. & F. $a$. & G. $a$. & P. a. & C. $p$. & C.s. & F.a. & G. $a$. & P. a. & Mean & SEM \\
\hline July & 7.4 & 6.1 & 3.9 & 2.4 & 12.6 & 4.9 & 6.7 & 3.8 & 3.4 & 7.6 & 5.9 & 0.2 \\
\hline August & 4.5 & 6.7 & 3.2 & 2.6 & 12.9 & 5.5 & 5.5 & 3.7 & 4.9 & 7.8 & 5.7 & 0.2 \\
\hline September & 4.6 & 6.9 & 4.0 & 2.7 & 8.0 & 4.9 & 7.8 & 4.4 & 3.5 & 8.1 & 5.5 & 0.2 \\
\hline October & 7.7 & 10.4 & 5.3 & 3.2 & 13.3 & 5.7 & 6.4 & 5.1 & 3.2 & 8.4 & 6.9 & 0.2 \\
\hline November & 5.6 & 10.8 & 5.5 & 3.2 & 12.7 & 6.7 & 6.4 & 6.0 & 6.1 & 6.0 & 6.9 & 0.2 \\
\hline December & 4.3 & 12.8 & 4.0 & 2.6 & 7.6 & 5.0 & 6.0 & 4.0 & 4.2 & 4.2 & 5.5 & 0.2 \\
\hline January & 4.7 & 9.7 & 3.5 & 2.2 & 9.0 & 4.5 & 6.1 & 3.7 & 5.5 & 4.2 & 5.3 & 0.2 \\
\hline February & 7.0 & 9.7 & 5.8 & 2.0 & 9.3 & 4.2 & 5.3 & 7.3 & 4.6 & 3.7 & 5.9 & 0.2 \\
\hline March & 3.8 & 11.1 & 5.7 & 7.0 & 9.2 & 7.3 & 7.8 & 6.8 & 10.6 & 6.7 & 7.6 & 0.2 \\
\hline April & 7.9 & 10.6 & 4.8 & 5.0 & 9.4 & 9.3 & 9.1 & 5.0 & 7.1 & 7.3 & 7.5 & 0.2 \\
\hline May & 6.0 & 8.8 & 4.2 & 3.7 & 10.4 & 8.7 & 8.7 & 4.0 & 7.5 & 9.5 & 7.1 & 0.2 \\
\hline June & 7.4 & 7.9 & 3.8 & 3.4 & 10.5 & 7.5 & 6.9 & 4.0 & 5.6 & 9.6 & 6.7 & 0.2 \\
\hline Mean & 5.9 & 9.3 & 4.5 & 3.3 & 10.4 & 6.2 & 6.9 & 4.8 & 5.5 & 6.9 & 6.4 & \\
\hline SEM & 0.2 & 0.2 & 0.2 & 0.2 & 0.2 & 0.2 & 0.2 & 0.2 & 0.2 & 0.2 & & \\
\hline Site mean & \multicolumn{12}{|c|}{ 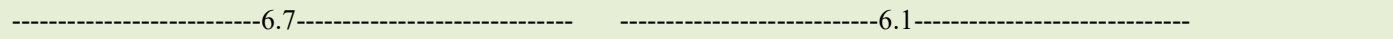 } \\
\hline SEM & \multicolumn{5}{|c|}{----------------------------0.1-------------------------------- } & \multicolumn{7}{|c|}{-----------------------------0.1------------------------------- } \\
\hline Factors & \multicolumn{2}{|c|}{---Month (A)---- } & Site (B) & \multicolumn{2}{|c|}{---Species (C)--- } & $\mathrm{A} \times \mathrm{B}$ & \multicolumn{2}{|c|}{-------A x C------ } & \multicolumn{2}{|c|}{--------B x C------ } & \multicolumn{2}{|c|}{---A x B x C-- } \\
\hline $\mathrm{p}$ & \multicolumn{2}{|c|}{-------" ${ }^{* * *}$--------- } & *** & \multicolumn{2}{|c|}{--------***------- } & *** & \multicolumn{2}{|c|}{ 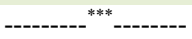 } & \multicolumn{2}{|c|}{--------"** --------- } & \multicolumn{2}{|c|}{------- ${ }^{* * *}-------$} \\
\hline
\end{tabular}

C. p.; Celtis pallida, C. s.; Croton suaveolens, F. a.; Forestiera angustifolia, G. a.; Guaiacum angustifolium, P. a.; Parkinsonia aculeata.

SEM, standard error of the mean.

${ }^{* * *}$ Significant at 0.001 level, ${ }^{* *}$ significant at 0.01 level. 
Table 7 - Monthly foliar Fe content ( $\mathrm{mg} \mathrm{kg}^{-1}$ dry matter) in five shrubs species from northeastern Mexico sampled at two study sites from July 2018 to June 2019. Data represents the mean $(\mathrm{n}=5)$.

\begin{tabular}{|c|c|c|c|c|c|c|c|c|c|c|c|c|}
\hline \multirow{2}{*}{$\begin{array}{l}\text { Site } \\
\text { Month }\end{array}$} & \multicolumn{5}{|c|}{ 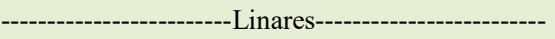 } & \multicolumn{5}{|c|}{ 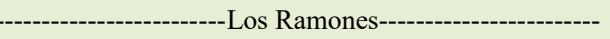 } & \multirow[b]{2}{*}{ Mean } & \multirow[b]{2}{*}{ SEM } \\
\hline & C. p. & C. s. & F.a. & G. $a$. & P. a. & C. p. & C. s. & F. $a$. & G. a. & P. a. & & \\
\hline July & 60.9 & 73.8 & 56.0 & 94.0 & 94.3 & 93.9 & 71.9 & 55.4 & 58.6 & 57.5 & 71.6 & 2.4 \\
\hline August & 74.8 & 92.3 & 50.9 & 94.0 & 99.3 & 81.0 & 89.7 & 72.1 & 94.1 & 70.4 & 81.8 & 2.4 \\
\hline September & 74.8 & 142.6 & 74.6 & 92.1 & 121.6 & 63.8 & 109.4 & 79.4 & 83.2 & 68.3 & 90.9 & 2.4 \\
\hline October & 56.4 & 59.2 & 67.7 & 113.6 & 124.7 & 63.6 & 64.8 & 79.9 & 81.2 & 69.3 & 78.0 & 2.4 \\
\hline November & 75.9 & 59.8 & 65.2 & 102.3 & 99.6 & 61.2 & 95.6 & 60.6 & 97.3 & 48.1 & 76.6 & 2.3 \\
\hline December & 72.1 & 76.0 & 68.1 & 94.9 & 125.3 & 63.7 & 129.0 & 54.5 & 89.6 & 53.8 & 82.7 & 2.4 \\
\hline January & 71.0 & 136.2 & 94.7 & 80.5 & 116.2 & 75.8 & 176.6 & 96.1 & 122.0 & 75.3 & 104.4 & 2.6 \\
\hline February & 66.8 & 127.9 & 72.5 & 91.5 & 112.3 & 78.7 & 171.4 & 112.6 & 108.8 & 75.3 & 101.8 & 2.6 \\
\hline March & 99.6 & 97.5 & 65.9 & 130.1 & 71.5 & 109.7 & 135.5 & 85.2 & 112.9 & 78.5 & 98.6 & 2.5 \\
\hline April & 65.6 & 96.2 & 43.9 & 86.2 & 74.1 & 93.1 & 150.1 & 68.3 & 103.0 & 68.6 & 84.9 & 2.3 \\
\hline May & 75.2 & 146.1 & 64.7 & 93.6 & 120.9 & 94.4 & 117.0 & 71.1 & 96.8 & 83.4 & 96.3 & 2.3 \\
\hline June & 74.4 & 66.2 & 58.7 & 139.1 & 97.3 & 59.5 & 48.5 & 44.1 & 137.8 & 65.1 & 79.1 & 2.3 \\
\hline Mean & 72.3 & 97.8 & 65.2 & 101.0 & 104.8 & 78.2 & 113.3 & 73.3 & 98.8 & 67.8 & 87.2 & \\
\hline SEM & 2.0 & 2.0 & 2.1 & 2.0 & 2.0 & 2.0 & 2.1 & 2.0 & 2.0 & 1.9 & & \\
\hline Site mean & \multicolumn{5}{|c|}{-------------------------88.2--------------------------- } & \multicolumn{5}{|c|}{-------------------------------86.3----------------------------- } & & \\
\hline SEM & \multicolumn{5}{|c|}{--------------------------0.9---------------------------- } & \multicolumn{5}{|c|}{ 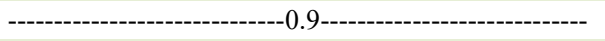 } & & \\
\hline Factors & \multirow{2}{*}{\multicolumn{2}{|c|}{---Month (A)--- }} & Site $(B)$ & \multicolumn{2}{|c|}{---Species (C)--- } & $\mathrm{A} \times \mathrm{B}$ & \multirow{2}{*}{\multicolumn{2}{|c|}{------A x C------- }} & -------B & C------ & \multirow{2}{*}{\multicolumn{2}{|c|}{$\begin{array}{c}--\mathrm{A} \times \mathrm{B} \times \mathrm{C}-- \\
---\cdot * *\end{array}$}} \\
\hline $\mathrm{p}$ & & & NS & -------- & ------- & *** & & & --------- & ------- & & \\
\hline
\end{tabular}

C. p.; Celtis pallida, C. s.; Croton suaveolens, F. a.; Forestiera angustifolia, G. a.; Guaiacum angustifolium, P. a.; Parkinsonia aculeata.

SEM, standard error of the mean.

${ }^{* * *}$ Significant at 0.001 level, ${ }^{* *}$ significant at 0.01 level, NS, Not significant.

requirements of mature small ruminants; $2.7,2.8$ and $2.6 \mathrm{~g} \mathrm{~kg}^{-1} \mathrm{DM}$ of $\mathrm{P}$, for sheep, goats and white-tailed deer, severally by NRC (2007) and indicated that there is a problem with the source and use of phosphorus. The low $\mathrm{P}$ content in native plants that grow in semiarid regions of northern Mexico was also reported by GUERRERO-CERVANTES et al. (2012), RAMÍREZ et al. (2010) and DOMÍNGUEZ-GÓMEZ et al. (2014). Thus, according to this situation phosphorus supplementation for small ruminants in semi-arid regions to meet their requirements its recommends by MAHMOOD et al. (2014).

The obtained results derived in a $\mathrm{Ca}: \mathrm{P}$ ratio of 40:1, was higher compared to wide $\mathrm{Ca}: \mathrm{P}$ ratios reported by RAMÍREZ et al. (2010). According to WILKENS et al. (2012), there are differences among ruminant species in the mechanisms to maintain $\mathrm{Ca}$ homeostasis, whereas small ruminants have mechanisms that help them to adapt diets with low $\mathrm{P}$ levels (HUBER et al., 2002). Apparently, goats, sheep and white-tailed deer can adapt to high proportions of Ca:P without being affected in their metabolism (RAMÍREZ, 1999).
Although, the content of $\mathrm{K}$ (total mean = $15.4 \mathrm{~g} \mathrm{~kg}^{-1} \mathrm{DM}$ ) varied between sites, species and months, this macronutrient was present in enough quantities to meet the growth requirements $(6.5,4.6$ and $4.6 \mathrm{~g} \mathrm{~kg}^{-1} \mathrm{DM}$ for $\mathrm{K}$ ), for goats, sheep, and whitetailed deer, respectively by NRC (2007), except $G$. angustifolium (4.1 $\mathrm{g} \mathrm{kg}^{-1} \mathrm{DM}$ ) during October and November in Linares site. In the present study, the foliar content of $\mathrm{K}$ was generally three times the requirement for small ruminants. These results are consistent with those reported by DERERO \& KITAW (2018), where potassium was the macromineral with the second highest concentration after $\mathrm{Ca}$ in fodder tree species in pastoral and agropastoral areas in eastern Ethiopia. DOMÍNGUEZGÓMEZ et al. (2014), reported similar results in semi-arid regions in northeastern of Mexico and HABIB et al. (2016), in arid and semi-arid rangelands of northern Pakistan and suggested that sodium supplementation, could avoid deficiencies caused by high concentrations of $\mathrm{K}$.

All species, except $P$. aculeata $\left(1.4 \mathrm{~g} \mathrm{~kg}^{-1}\right.$ $\mathrm{DM})$ in Los Ramones site and F. angustifolia (1.2 g 
Table 8 - Monthly foliar Mn content ( $\mathrm{mg} \mathrm{kg}^{-1}$ dry matter) in five shrubs species from northeastern Mexico sampled at two study sites from July 2018 to June 2019. Data represents the mean $(n=5)$.

\begin{tabular}{|c|c|c|c|c|c|c|c|c|c|c|c|c|}
\hline \multirow{2}{*}{$\begin{array}{l}\text { Site } \\
\text { Month }\end{array}$} & \multicolumn{5}{|c|}{---------------------------Linares-------------------------- } & \multicolumn{7}{|c|}{ 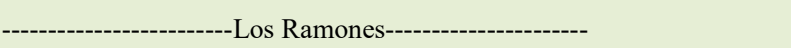 } \\
\hline & C.p. & C.s. & F. $a$. & G. $a$. & P. a. & C.p. & C.s. & $F . a$. & G. $a$. & P. a. & Mean & SEM \\
\hline July & 34.7 & 13.5 & 44.2 & 30.4 & 57.6 & 32.4 & 24.1 & 19.6 & 27.4 & 23.8 & 30.8 & 0.9 \\
\hline August & 34.8 & 12.1 & 30.7 & 28.4 & 40.1 & 25.3 & 18.3 & 22.3 & 34.0 & 39.3 & 28.5 & 0.9 \\
\hline September & 43.7 & 13.1 & 38.4 & 40.1 & 55.4 & 35.9 & 25.1 & 24.3 & 32.6 & 52.5 & 36.1 & 0.9 \\
\hline October & 26.4 & 21.5 & 57.3 & 34.5 & 48.2 & 44.9 & 22.1 & 29.2 & 26.2 & 32.8 & 34.3 & 0.9 \\
\hline November & 22.9 & 18.8 & 78.6 & 35.6 & 49.6 & 29.8 & 16.7 & 19.6 & 32.0 & 25.3 & 32.9 & 1.0 \\
\hline December & 32.8 & 28.3 & 78.9 & 47.9 & 64.2 & 29.3 & 25.3 & 36.9 & 51.3 & 38.1 & 43.3 & 0.9 \\
\hline January & 25.9 & 25.9 & 54.3 & 30.9 & 70.3 & 45.7 & 27.4 & 46.6 & 48.7 & 38.9 & 41.4 & 1.0 \\
\hline February & 32.9 & 26.2 & 58.3 & 36.3 & 64.6 & 35.8 & 22.5 & 46.0 & 59.2 & 48.1 & 43.0 & 1.0 \\
\hline March & 52.5 & 23.4 & 50.0 & 36.3 & 31.8 & 33.3 & 22.4 & 26.4 & 26.6 & 31.0 & 33.4 & 0.9 \\
\hline April & 32.4 & 21.1 & 37.8 & 31.1 & 40.9 & 24.2 & 28.4 & 22.4 & 21.6 & 33.2 & 29.3 & 0.9 \\
\hline May & 33.6 & 23.8 & 33.6 & 31.4 & 56.6 & 27.0 & 28.0 & 25.1 & 34.4 & 43.8 & 33.8 & 0.9 \\
\hline June & 29.2 & 18.5 & 37.9 & 41.1 & 59.9 & 36.6 & 28.1 & 32.3 & 47.0 & 55.9 & 38.6 & 0.9 \\
\hline Mean & $33.5^{*}$ & 20.5 & 50.0 & $35.3 *$ & 53.3 & $33.3^{*}$ & 24.0 & 29.2 & $36.7 *$ & 38.6 & 35.5 & \\
\hline SEM & 0.8 & 0.8 & 0.9 & 0.8 & 0.9 & 0.8 & 0.8 & 0.8 & 0.8 & 0.8 & & \\
\hline Site mean & \multicolumn{5}{|c|}{ 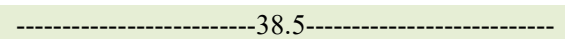 } & \multicolumn{7}{|c|}{ 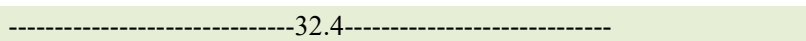 } \\
\hline SEM & \multicolumn{5}{|c|}{ 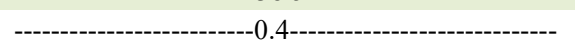 } & \multicolumn{7}{|c|}{ 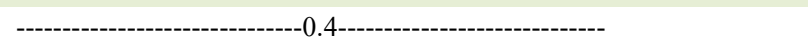 } \\
\hline Factors & \multicolumn{2}{|c|}{---Month (A)--- } & Site (B) & \multicolumn{2}{|c|}{---Species (C)--- } & $\mathrm{A} \times \mathrm{B}$ & \multicolumn{2}{|c|}{-------A x C------ } & \multicolumn{2}{|c|}{--------B x C------- } & \multicolumn{2}{|c|}{--A x B x C-- } \\
\hline $\mathrm{p}$ & \multicolumn{2}{|c|}{-------- ${ }^{* * *}$} & $* * *$ & \multicolumn{2}{|c|}{------ ${ }^{* * *}$} & $* * *$ & \multicolumn{2}{|c|}{--------"** -------- } & \multicolumn{2}{|c|}{--------"** -------- } & \multicolumn{2}{|c|}{------- ${ }^{* * *}-----$} \\
\hline
\end{tabular}

C. p.; Celtis pallida, C. s.; Croton suaveolens, F. a.; Forestiera angustifolia, G. a.; Guaiacum angustifolium, P. a.; Parkinsonia aculeata.

SEM, standard error of the mean.

${ }^{* * *}$ Significant at 0.001 level, ${ }^{* *}$ significant at 0.01 level. ${ }^{*}$ Site factor were not significant between same shrub species.

$\left.\mathrm{kg}^{-1} \mathrm{DM}\right)$ in Linares site during February and May, respectively, contained enough foliar $\mathrm{Mg}$ (total mean $=3.7 \mathrm{~g} \mathrm{~kg}^{-1} \mathrm{DM}$ ) throughout the year to meet the metabolic requirements by NRC (2007) of adult sheep ( $1.5 \mathrm{~g} \mathrm{~kg}^{-1} \mathrm{DM}$ of $\left.\mathrm{Mg}\right)$. Otherwise, $P$. aculeata (1.5 $\left.\mathrm{g} \mathrm{kg}^{-1} \mathrm{DM}\right)$ in both sites and $F$. angustifolia (1.5 $\left.\mathrm{g} \mathrm{kg}^{-1} \mathrm{DM}\right)$ in Linares site during April showed insufficient levels to meet requirements for goats and white-tailed deer (1.6 $\mathrm{g} \mathrm{kg}^{-1} \mathrm{DM}$ of Mg). Similar results were reported by RAMÍREZ et al. (2001) in 14 native shrubs of northeastern Mexico. RAMÍREZ et al. (2010) and DOMÍNGUEZ-GÓMEZ et al. (2014) for 6 and 4 native shrubs of northeastern Mexico, respectively, also argued that the low $\mathrm{Mg}$ content in winter months could be due to the low temperatures. In the present study, the average temperature during December and February ranged from $14.3^{\circ} \mathrm{C}$ to 18.2 ${ }^{\circ} \mathrm{C}$. These lowest monthly temperatures recorded, could explain why the lowest $\mathrm{Mg}$ levels were found in these months during the study.

In general, the studied species contained insufficient amounts of foliar $\mathrm{Cu}$ (total mean $=6.4 \mathrm{mg}$ $\left.\mathrm{kg}^{-1} \mathrm{DM}\right)$. However, adequate contents were present unevenly in all the shrubs species throughout the year. The species $C$. suaveolens (Linares site mean $=9.3 \mathrm{mg}$ $\mathrm{kg}^{-1} \mathrm{DM}$ ) and $P$. aculeata (Linares site mean $=10.4 \mathrm{mg}$ $\mathrm{kg}^{-1} \mathrm{DM}$ ) showed sufficient levels of foliar $\mathrm{Cu}$ to meet the requirements of small ruminants in adult range (9.0 $\mathrm{mg} \mathrm{kg}^{-1} \mathrm{DM}$ of $\mathrm{Cu}$, for sheep, goats and whitetailed deer), in general by NRC (2007). This result agreed with reports of RAMÍREZ et al. (2010) and GUERRERO-CERVANTES et al. (2012), in native species consumed by small ruminants in semi-arid regions of northeastern and northern Mexico, during the spring months. RAMÍREZ et al. (2001) explained that the highest $\mathrm{Cu}$ content observed is based on the seasonal pattern of plant growth, about which growth rates appeared to be higher during the spring season in this region. According to RAMÍREZ-ORDUÑA et al. (2005), low $\mathrm{Cu}$ levels in shrubs might be caused because the high $\mathrm{pH}$ of soil in semi-arid regions of Baja California Sur, Mexico.

In all shrub species studied, Fe (total mean $=87.2 \mathrm{mg} \mathrm{kg}^{-1} \mathrm{DM}$ ) concentrations exceeded the requirements by NRC (2007), for grazing ruminants $\left(50 \mathrm{mg} \mathrm{kg}^{-1} \mathrm{DM}\right.$ of $\mathrm{Fe}$, for sheep, goats 
Table 9 - Monthly foliar $\mathrm{Zn}$ content ( $\mathrm{mg} \mathrm{kg}^{-1}$ dry matter) in five shrubs species from northeastern Mexico sampled at two study sites from July 2018 to June 2019. Data represents the mean $(\mathrm{n}=5)$.

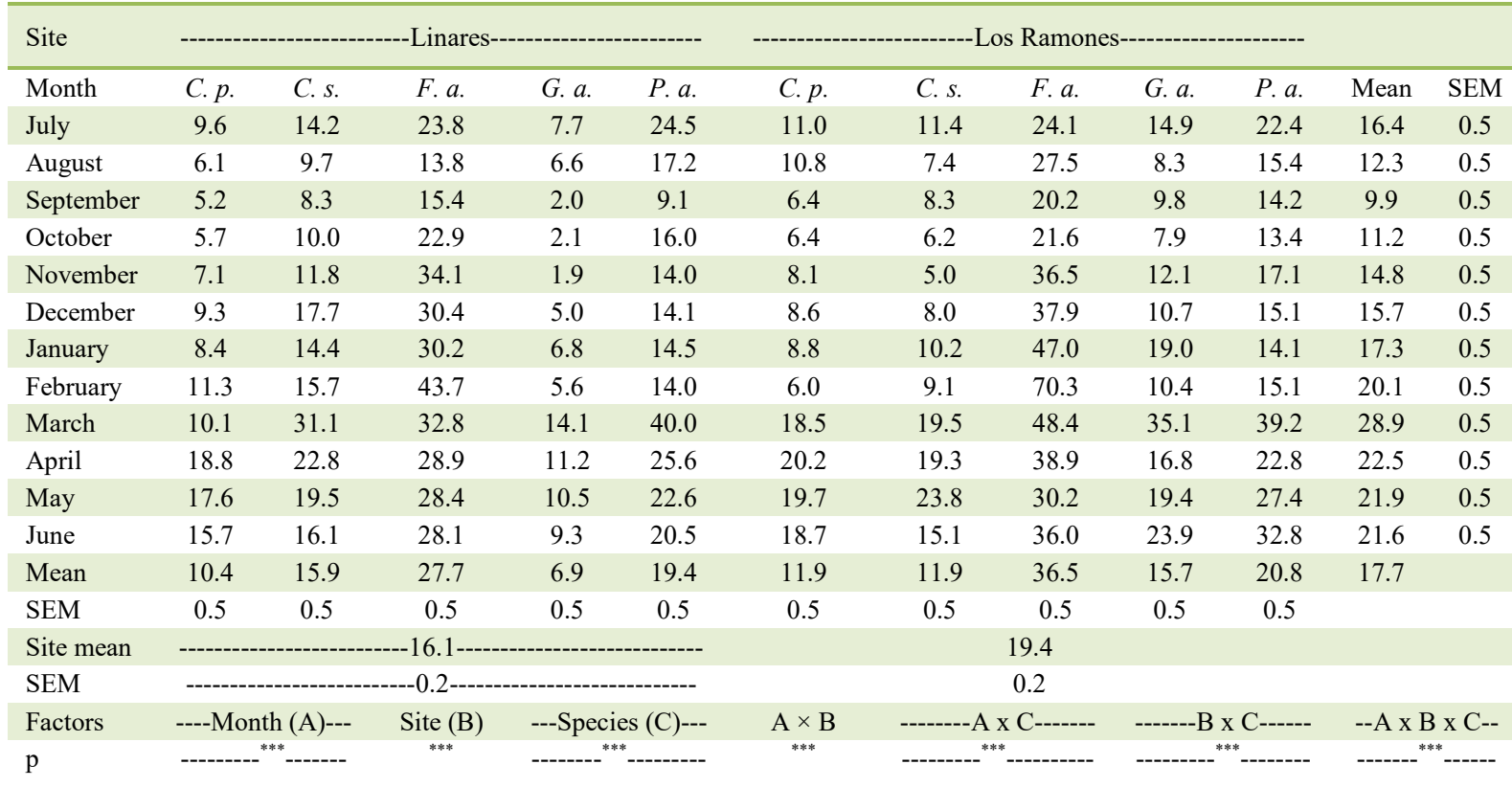

C. p.; Celtis pallida, C. s.; Croton suaveolens, F. a.; Forestiera angustifolia, G. a.; Guaiacum angustifolium, P. a.; Parkinsonia aculeata.

SEM, standard error of the mean.

Significant at 0.001 level, ${ }^{* * *}$ significant at 0.01 level.

and white-tailed deer). Satisfactory Iron levels for small ruminants have been reported by RAMÍREZORDUÑA et al. (2005), GUERRERO-CERVANTES et al. (2012) in northern Mexico and by HABIB et al. (2016) in semi-arid rangelands of Pakistan.

All species, with the exception of $C$. suaveolens (Linares site mean $=20.5 \mathrm{Mn} \mathrm{mg} \mathrm{kg-1}$ $\mathrm{DM}$ and Los Ramones site mean $=24.0 \mathrm{Mn} \mathrm{mg} \mathrm{kg}{ }^{-1}$ $\mathrm{DM}$ ), contain enough $\mathrm{Mn}$ content in leaves (total mean $=35.5 \mathrm{mg} \mathrm{kg}^{-1}$ ) to meet the requirements by NRC (2007) of adult small ruminants $\left(30 \mathrm{mg} \mathrm{kg}^{-1}\right.$ $\mathrm{DM}$ of $\mathrm{Mn}$, for sheep, goats and white-tailed deer). GUERRERO-CERVANTES et al. (2012), reported low levels of $\mathrm{Mn}$ in shrub leaves of northern Mexico. The low level of Mn in August in this study could be associate to dry season, also reported for native small trees and shrubs growing in different rangelands of Mexico by RAMÍREZ et al. (2006). Also, the high foliar content of $\mathrm{Ca}$ may increase $\mathrm{Mn}$ requirements, due to the probable obstruction of $\mathrm{Mn}$ absorption (RAMÍREZ et al., 2010).

The shrub species of this study did not show consistent concentrations of $\mathrm{Zn}$ (total mean = $\left.17.7 \mathrm{mg} \mathrm{kg}^{-1} \mathrm{DM}\right)$ to meet the requirements by NRC (2007), for adult small ruminants $(40,45$ and $45 \mathrm{mg}$ $\mathrm{kg}^{-1} \mathrm{DM}$ of $\mathrm{Zn}$, for sheep, goats and white-tailed deer, separately). Acceptable concentrations for sheep were registered in F. angustifolia in the months of January (Los Ramones site $47.0 \mathrm{mg} \mathrm{kg}^{-1} \mathrm{DM}$ ), February (Linares site $=43.7 \mathrm{mg} \mathrm{kg}^{-1} \mathrm{DM}$ and Los Ramones site $=70.3 \mathrm{mg}$ $\mathrm{kg}^{-1} \mathrm{DM}$ ) and March (Los Ramones site $=48.4 \mathrm{mg} \mathrm{kg}^{-1}$ DM). In the case of $P$. aculeata, only in March (Linares site $40.0 \mathrm{mg} \mathrm{kg}^{-1} \mathrm{DM}$ ) acceptable values were registered for sheep, according to our results March showed the highest levels of $\mathrm{Zn}$, previous studies showed that this situation generally occurred in tropical shrubs during spring season (HASSEN et al., 2007). Similar levels of Zn were reported by RAMÍREZ et al. (2010) and DOMÍNGUEZ-GÓMEZ et al. (2014) in native plants of northeastern Mexico. These authors argued that only some species can meet the requirements of adult ruminants during certain seasons of the year. In the same way, HABIB et al. (2016) reported that only certain species contain adequate levels of $\mathrm{Zn}$ for grazing small ruminants in semi-arid regions of northern Pakistan.

Ciência Rural, v.50, n.10, 2020. 


\section{CONCLUSION}

The species $C$. pallida showed the best levels of macro-minerals, while $P$. aculeata had the highest levels of micro-minerals. The shrub species in this study can be considered a good source of $\mathrm{Ca}, \mathrm{K}, \mathrm{Mg}, \mathrm{Fe}$ and $\mathrm{Mn}$ except in C. suaveolens. However, $\mathrm{P}, \mathrm{Cu}$ and $\mathrm{Zn}$ must be supplemented to meet the metabolic requirements of small ruminants in extensive grazing, in areas where they consume these shrubs. Furthermore, feeding and silvopastoralagroforestry studies are required to select the best shrub species to ensure its efficient adaptability and acceptance by small ruminants in semiarid rangeland of northeastern Mexico.

\section{ACKNOWLEDGMENTS}

Authors gratefully thank to Ph.D. Roque Gonzalo Ramirez Lozano $(\dagger)$. Also, the valuable technical assistance provided by Mrs. Elsa Dolores González Serna and Mr. Manuel Hernández Charles is gratefully recognized. We also thankfully acknowledge to Consejo Nacional de Ciencia y Tecnología (CONACYT) for financial support of this research by Project Grant No. 250732, (Convocatoria de Investigación Ciencia Básica, 2015) and providing a doctoral scholarship to the first author.

\section{DECLARATION OF CONFLICT OF INTERESTS}

The authors state that there is no conflict of interest with any financial organization regarding the material discussed in the manuscript.

\section{AUTHORS' CONTRIBUTIONS}

HGR and MCE conceived and designed experiments MCE performed the experiments, carried out the lab analyses and performed statistical analyses of experimental data. MVGM supervised statistical analyzes of experimental data. MCE, ICS, HBB and HGR prepared the draft of the manuscript. All authors critically revised the manuscript and approved of the final version.

\section{REFERENCES}

AOAC Official methods of analysis of AOAC International. 16th ed. Washington, D.C., 1997.

CANTÚ, I. et al. Caracterización fisicoquímica de un calcisol bajo diferentes sistemas de uso de suelo en el noreste de México. Revista Mexicana de Ciencias Forestales, v.9, n.49, p.59-86, 2018. Available from: <https://cienciasforestales.inifap.gob.mx/ editorial/index.php/forestales/article/view/153/1664>. Accessed: Apr. 15, 2020. doi: 10.29298/rmcf.v9i49.153.

DERERO, A; KITAW, G. Nutritive values of seven high priority indigenous fodder tree species in pastoral and agro-pastoral areas in Eastern Ethiopia. Agriculture and Food Security, v.7, n.1, p.19, 2018. Available from: <http://www.agricultureandfoodsecurity. biomedcentral.com/articles>. Accessed: Feb. 04, 2020. doi: 10.1186/s40066-018-0216-y.

DOMÍNGUEZ-GÓMEZ, T. G. et al. Mineral content in four browse species from northeastern Mexico. Pakistan Journal of Botany, v.46, n.4, p.1421-1429, 2014. Available from: <https:// www.researchgate.net/publication/264649325_Mineral_content in_four_browse_species_from_northeastern_Mexico $>$. A Accessed: Feb. 04, 2020.

DOMÍNGUEZ-GÓMEZ, T. G. et al. Importancia nutrimental en plantas forrajeras del matorral espinoso tamaulipeco. Ciencia UANL, v.15, n.59, p.77-93, 2012. Available from: <https:// www.researchgate.net/publication/264041669_Importancia nutrimental_en_plantas_forrajeras_del_matorral_espinoso tamaulipeco>. Accessed: Feb. 04, 2020.

ESTELL, R. E. et al. Increasing shrub use by livestock in a world with less grass. Rangeland Ecology and Management, v.65, n.6, p.553-562, 2012. Available from: <https:/www.researchgate.net/ publication/260138625_Increasing_Shrub_Use_by_Livestock in a World with Less Grass>. Accessed: Feb. 04, 2020. doi: 10.2111/REM-D-11-00124.1.

ESTRADA-CASTILLÓN, E. et al. Ethnobotanical survey of useful species in Bustamante, Nuevo León, Mexico. Human Ecology, v.46, n.1, p.117-132, 2018. Available from: <https://www. researchgate.net/publication/321729079 Ethnobotanical Survey of_Useful_Species_in_Bustamante_Nuevo_Leon_Mexico>. Accessed: Feb. 04, 2020. doi: 10.1007/s10745-017-9962-x.

FAN, Q. et al. Relationship of mineral elements in sheep grazing in the highland agro-ecosystem. Asian-Australasian Journal of Animal Sciences, v.33, n.1, p.44-52, 2019. Available from: $<$ https://www.ajas.info/journal/view.php?number=24264>. Accessed: Apr. 15, 2020. doi: 10.5713/ajas.18.0955.

FOROUGHBAKHCH, R. Establishment and growth potential of fuelwood species in northeastern Mexico. Agroforestry Systems, v.19, n.2, p.95-108, 1992. Available from: <https:// www.researchgate.net/publication/225439280 Establishment and growth potential of fuelwood species in northeastern Mexico>. Accessed: Feb. 04, 2020. doi: 10.1007/BF00138500.

FOROUGHBAKHCH, R. et al. Use of quantitative methods to determine leaf biomass on 15 woody shrub species in northeastern Mexico. Forest Ecology and Management, v.216, n.1-3, p.359-366, 2005. Available from: <https://www.researchgate. net/publication $/ 248427840$ _ Use_of_quantitative_methods to_determine_leaf_biomass_on_15_woody_shrub_species_in northeastern_Mexico $>$. Accessed: Feb. 04, 2020. doi: 10.1016/j. foreco.2005.05.046.

GONZÁLEZ-RODRÍGUEZ, H. et al. Plant water relations of thornscrub shrub species, north-eastern Mexico. Journal of Arid Environments, v.58, n.4, p.483-503, 2004. Available from: <https://www.researchgate.net/publication/256941205 Plant_water_relations_of_thornscrub_shrub_species_northeastern Mexico $>$. Accessed: Feb. 04, 2020. doi: 10.1016/j. jaridenv.2003.12.001.

GUERRERO-CERVANTES, M. et al. Dry matter digestion of native forages consumed by range goats in North Mexico. Journal of Animal and Veterinary Advances, v.8, n.3, p.408-412, 2008. Available from: <https://www.researchgate. net/publication/285778139_Dry_Matter_Digestion_of_Native_ 
Forages_Consumed_by_Range_Goats_in_North_Mexico>. Accessed: Feb. 04, 2020

GUERRERO-CERVANTES, M. et al. Mineral content in range forages from north Mexico. Journal of Applied Animal Research, v.40, n.2, p.102-107, 2012. Available from: <https:// www.researchgate.net/publication/254215263 Mineral content in_range_forages_from_north_Mexico $>$. Accessed: Feb. 04, 2020. doi: 10.1080/09712119.2011.607907.

HABIB, G. et al. Nutritive value of common tree leaves for livestock in the semi-arid and arid rangelands of Northern Pakistan. Livestock Science, v.184, p.64-70, 2016. Available from: <https:// www.researchgate.net/publication/287966969_Nutritive_value of common tree leaves for livestock in the semi-arid and arid_rangelands_of_Northern_Pakistan>. Accessed: Feb. 04, 2020. doi: $10.1016 / \bar{j}$. livsci. 2015.12 .009 .

HASSEN, A. et al. Influence of season/year and species on chemical composition and in vitro digestibility of five Indigofer accessions. Animal Feed Science and Technology, v.136, n.3-4, p.312-322, 2007. Available from: <https://www.researchgate.net/ publication/248333214_Influence_of_seasonyear_and_species on chemical composition and in vitro digestibility of five Indigofera_accessions $>$. Accessed: Feb. 04,2020 . doi: $10.1016 / \bar{j}$. anifeedsci. 2006.09 .010 .

HUBER, K. et al. Phosphate transport in the duodenum and jejunum of goats and its adaptation by dietary phosphate and calcium. American Journal of Physiology - Regulatory Integrative and Comparative Physiology, v.283, n.2, p.296-302, 2002. Available from: <https:// www.researchgate.net/publication/11256817_Phosphate_transport_in the duodenum and jejunum of goats and its adaptation by dietary_phosphate_and_calcium $>$. Accessed: Feb. 04, 2020. doi: 10.1152/ajpregu.00760.2001

LUDWIG, J. A. et al. Size-biomass relationships of severa Chihuahuan Desert shrubs. American Midland Naturalist, v.94 n.2, p.451, 1975. Available from: <https://www.researchgate.net/ publication/253204022_Size6iomass_Relationships_of_Several Chihuahuan Desert_Shrubs $>$. Accessed: Feb. 04, 2020. doi: $10.2307 / 2424437$.

MAHMOOD, A. et al. Assessment of phosphorus levels in small ruminants as affected by summer and winter. Journal of Northeast Agricultural University (English Edition), v.21, n.1, p.45-51, 2014. Available from: <https://www.researchgate.net/ publication/274020105 Assessment of Phosphorus Levels in_Small_Ruminants_as_Affected_by_Summer_and_Winter>. Accessed: Feb. 04, 2020. doi: 10.1016/s10068104(14)60021-0.

MEYER, M. W. et al. Protein and energy content of whitetailed deer diets in the Texas Coastal Bend. Journal of Wildlife Management, v.48, n.2, p.527-534, 1984. Available from: $<$ https:// www.researchgate.net/publication/229192284 Protein and Energy_Content_of_WhiteTailed_Deer_Diets_in_the_Texas Coastal Bend>. Accessed: Feb. 04, 2020. doi: 10.2307/3801185.

NATIONAL RESEARCH COUNCIL. NRC, Nutrient requirements of small ruminants: sheep, goats, cervids, and new world camelids. National Academies, 2007. Washington, DC., USA.

RAMÍREZ, R. G. et al. White-tailed deer food habits in northeastern Mexico. Small Ruminant Research, v.25, n.2, p.141-146, 1997. Available from: <https://www.sciencedirect.com/science/article
abs/pii/S0921448896009601>. Accessed: Feb. 04, 2020. doi: 10.1016/S0921-4488(96)00960-1.

RAMÍREZ, R. G. Feed resources and feeding techniques of small ruminants under extensive management conditions. Small Ruminant Research, v.34, n.3, p.215-230, 1999. Available from: $<$ https://www. sciencedirect.com/science/article/abs/pii/S0921448899000759>. Accessed: Feb. 04, 2020. doi: 10.1016/S0921-4488(99)00075-9.

RAMÍREZ, R. G. et al. Seasonal variation of macro and trace mineral contents in 14 browse species that grow in northeastern Mexico. Small Ruminant Research, v.39, n.2, p.153-159, 2001. Available from: <https://www.sciencedirect.com/science/article/ abs/pii/S092144880000184X>. Accessed: Feb. 04, 2020. doi: 10.1016/S0921-4488(00)00184-X

RAMÍREZ, R. G. et al. Seasonal trends of macro and micro minerals in 10 browse species that grow in northeastern Mexico. Animal Feed Science and Technology, v.128, n.1-2, p.155-164, 2006. Available from: $<$ https://www.researchgate.net/publication/248332467 Seasonaltrends_of_macro_and_micro_minerals_in_10_browse species that grow in northeastern Mexico>. Accessed: Feb. 04, 2020. doi: $10.1016 / \bar{j}$.anifeedsci.2005.10.005.

RAMÍREZ, R. G. et al. Spatio-temporal variations of macro and trace mineral contents in six native plants consumed by small ruminants at Northeastern Mexico. Tropical and Subtropical Agroecosystems, v.12, n.2, p.267-281, 2010. Available from: $<$ https://www.academia.edu/32336564/Spatio-temporal variations of_macro and trace mineral_contents_in_six_native plants consumed by ruminants at northeastern mexico $>$. Accessed: Feb. 04, 2020.

RAMÍREZ-ORDUÑA, R. et al. Mineral content of browse species from Baja California Sur, Mexico. Small Ruminant Research, v.57, n.1, p.1-10, 2005. Available from: <https://www. researchgate.net/publication/278665555 Mineral content of browse_species_from_Baja_California_Sur_Mexico $>$. Accessed: Feb. 04, 2020. doi: 10.1016/j.smallrumres.2004.03.004.

SALEM, A. Z. M. et al. Effect of increasing levels of seven tree species extracts added to a high concentrate diet on in vitro rumen gas output. Animal Science Journal, v.85, n.9, p.853-860, 2014. Available from: $<$ https://onlinelibrary.wiley.com/doi/full/10.1111/ asj.12218>. Accessed: Feb. 04, 2020. doi: 10.1111/asj.12218.

SANON, H. O. et al. Behaviour of goats, sheep and cattle and their selection of browse species on natural pasture in a Sahelian area. Small Ruminant Research, v.67, n.1, p.64-74, 2007. Available from: <https://www.sciencedirect.com/science/article/pii/ S0921448805003846>. Accessed: Feb. 04, 2020. doi: 10.1016/j. smallrumres.2005.09.025.

SCHINDLER, J. R. et al. Influence of thorns and tannins on whitetailed deer browsing after mowing. Journal of Arid Environments, v.55, n.2, p.361-377, 2003. Available from: <https://www. sciencedirect.com/science/article/abs/pii/S0140196303000211>. Accessed: Feb. 04, 2020. doi: 10.1016/S0140-1963(03)00021-1.

WILKENS, M. R. et al. In contrast to sheep, goats adapt to dietary calcium restriction by increasing intestinal absorption of calcium. Comparative Biochemistry and Physiology - A Molecular and Integrative Physiology, v.163, n.3-4, p.396-406, 2012. Available from: <https://www.sciencedirect.com/science/article/ pii/S1095643312003686?via\%3Dihub>. Accessed: Feb. 04, 2020. doi: 10.1016/j.cbpa.2012.06.011. 